\title{
Study of the Effect of Different Hole Sizes on Mechanical Strength of Wafers for Back Contact Solar Cells
}

\author{
E. Cereceda ${ }^{1}$, J. Barredo ${ }^{2}$, J.R. Gutiérrez ${ }^{1}$, J.C. Jimeno ${ }^{1}$, A. Fraile ${ }^{3}$ and L. Hermanns ${ }^{3}$ \\ ${ }^{1}$ Technological Institute of Microelectronics (TiM), University of the Basque Country, Zamudio, Spain \\ ${ }^{2}$ Centre for Modeling in Mechanical Engineering (CEMIM-F2I2), Madrid, Spain \\ ${ }^{3}$ Department of Structural Mechanics and Industrial Constructions, UPM, Madrid, Spain
}

\begin{abstract}
Drilling process on wafers to produce EWT or MWT solar cells is a critical fabrication step, which affects on their mechanical stability. The amount of damage introduced during drilling process depends on the density of holes, their size and the chemical process applied afterwards. To quantify the relation between size of the holes and reduction of mechanical strength, several sets of wafers have been prepared, with different hole diameter. The mechanical strength of these sets has been measured by the ring on ring bending test, and the stress state in the moment of failure has been deduced by FE simulation.
\end{abstract}

Index Terms - back contact, photovoltaic cells, silicon, stress measurement, surface cracks.

\section{INTRODUCTION}

Back contact silicon solar cells have some fabrication steps that modify its mechanical structure. Emitter wrap through (EWT) solar cell has tiny holes over the whole area and metal wrap through (MWT) cell has fewer but bigger holes. Due to these holes, both positive and negative contacts can be placed on the back side of the wafer, increasing the effective area of the cell and simplifying the interconnection process.

Apart of these advantages, the generation of a drilled structure has an important disadvantage relating to mechanical properties of the cell. The presence of holes reduces significantly the strength of these wafers [1-4] increasing the breakage ratio in the cell production line. This ratios are critical for the PV industry [5,6] and, therefore, studies concerning the influence of the holes in the mechanical strength of these type of wafers are gaining popularity last years [1-4].

These holes act as stress concentrators reducing mechanical strength of the wafers. Some factors have been observed that affects this reduction. An increase on the hole density involves that distance between neighboring holes is reduced. If two holes are close enough, their stress concentration zones can overlap, leading to a greater part of the surface subjected to a higher stress [4].

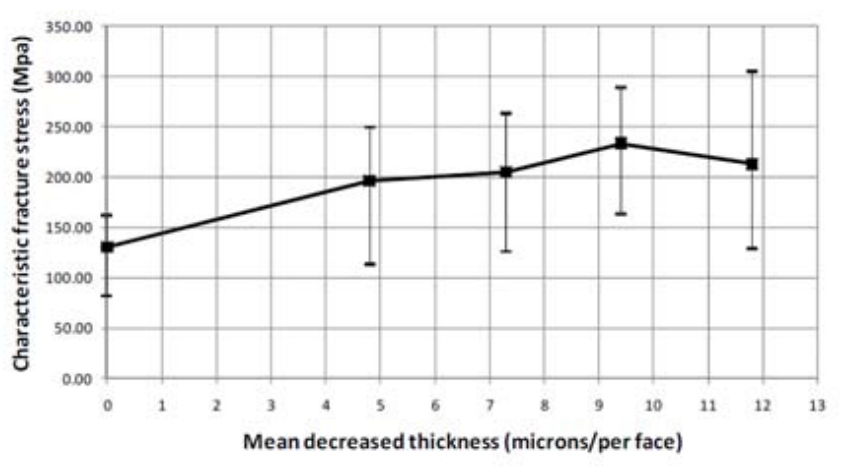

Fig. 1. Characteristic fracture stress versus decreased thickness per face.

Moreover, it's necessary to take into account the damage induced by the drilling process. It has been proved that a subsequent chemical bath can partially remove the laser damage $[1,3]$. It has also been observed that, as the etching process time increases, also increases the characteristic fracture stress of the wafer. However, it seems that if the processing time continues increasing, the characteristic fracture stress begins to drop (see Fig.1).

The chemical etching process reducing the thickness is also enlarging the holes diameter. Therefore, these baths have two opposite effects: on one hand, the bath is removing the microcracks coming from the wire sawing and laser drilling processes; on the other hand, the bath is increasing the diameter and as a consequence, the stress concentration factor. This may justify the curve observed in figure 1 . This work is focused on the hole size effect on mechanical strength of the wafer.

\section{PREPARATION OF SAMPLES}

To carry on this study, three sets of $125 \times 125 \mathrm{~mm}$ pseudosquare monocrystalline silicon wafers are prepared. All the wafers are subjected to an etching process prior to the drilling step, in order to remove any possible wiresaw surface damage. This etching is 2 minutes long and removes $7 \mu \mathrm{m}$ per face.

The next step is the drilling process, in witch holes are made in the wafers by laser. The hole distribution is the same in 
every wafer. It consist on a matrix of $15 \times 15$ holes with a distance between hole centers of $2 \mathrm{~mm}$ in both directions. The laser drilling process is performed with a $\mathrm{Q}$ switched fiber laser from EOLITE Systems (France), which generates pulses of $10 \mathrm{~ns}$, working in a wavelength of $515 \mathrm{~nm}$.

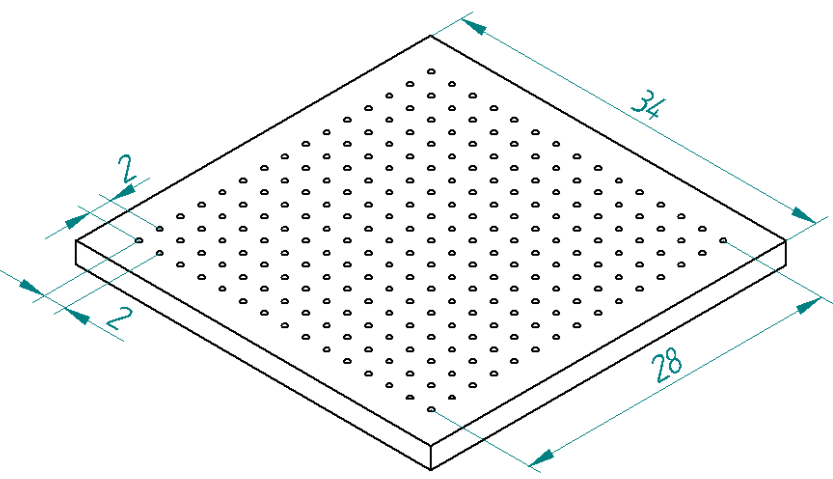

Fig. 2. Dimensions of the samples.

In order to remove the damage generated during the laser process, a final chemical etching is applied to the wafers. This final bath is longer and removes $12.5 \mu \mathrm{m}$ per face. Final thickness of the samples remains between $160 \mu \mathrm{m}$ and $170 \mu \mathrm{m}$. Processing the wafers in that way, the only difference from set to set is the diameter of the holes. The hole diameter are approximately; $60 \mu \mathrm{m}$ for set 1 and $140 \mu \mathrm{m}$ for set 2 . The third set of wafers, named as set 0 , remains without holes and it is used as reference.

Finally, the wafers are cut to get 9 samples of $34 \mathrm{~mm} \times 34$ $\mathrm{mm}$ from each wafer. Samples dimensions are shown in Fig. 2.
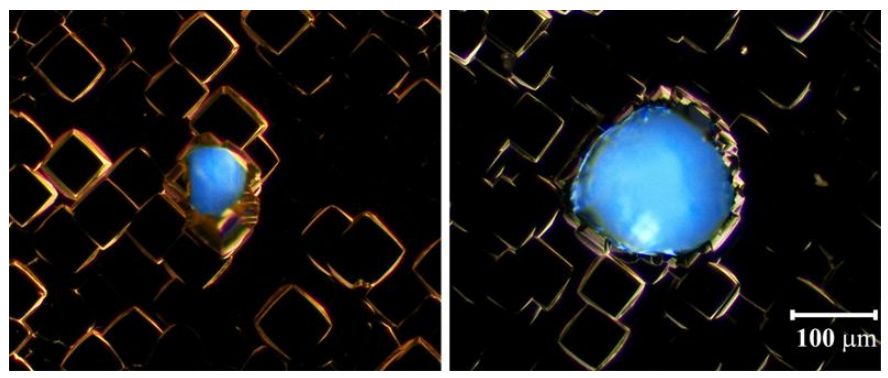

Fig. 3. Hole appearance for sets 1 and 2.

\section{RING ON RING TEST}

The Ring on Ring bending test is chosen to evaluate the surface damage induced by the holes. In this test, the wafer is supported on a ring of $20 \mathrm{~mm}$ of diameter and a controlled load is applied on the front side by an upper ring of $10 \mathrm{~mm}$ of diameter.

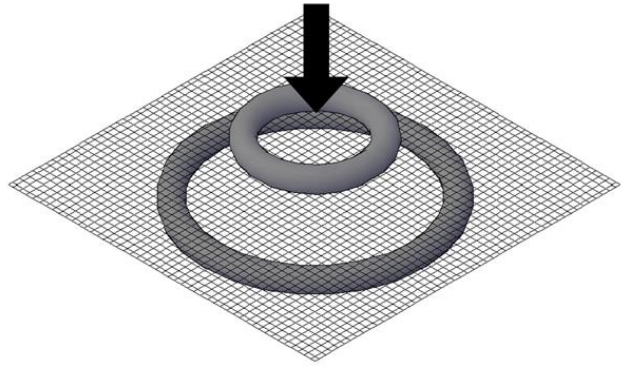

Fig. 4. Schematic of the Ring on Ring bending test.

This test is appropriate for this study because tensions within the lower ring are much larger than the outer part [7]. Therefore, the evaluation of the strength takes into account only the damage caused by the holes, neglecting the influence of any existing damage on the edges of the wafer.

\section{NUMERICAL MODEL}

The information we get from the test is the behavior of the samples and the maximum load and displacement before the failure.
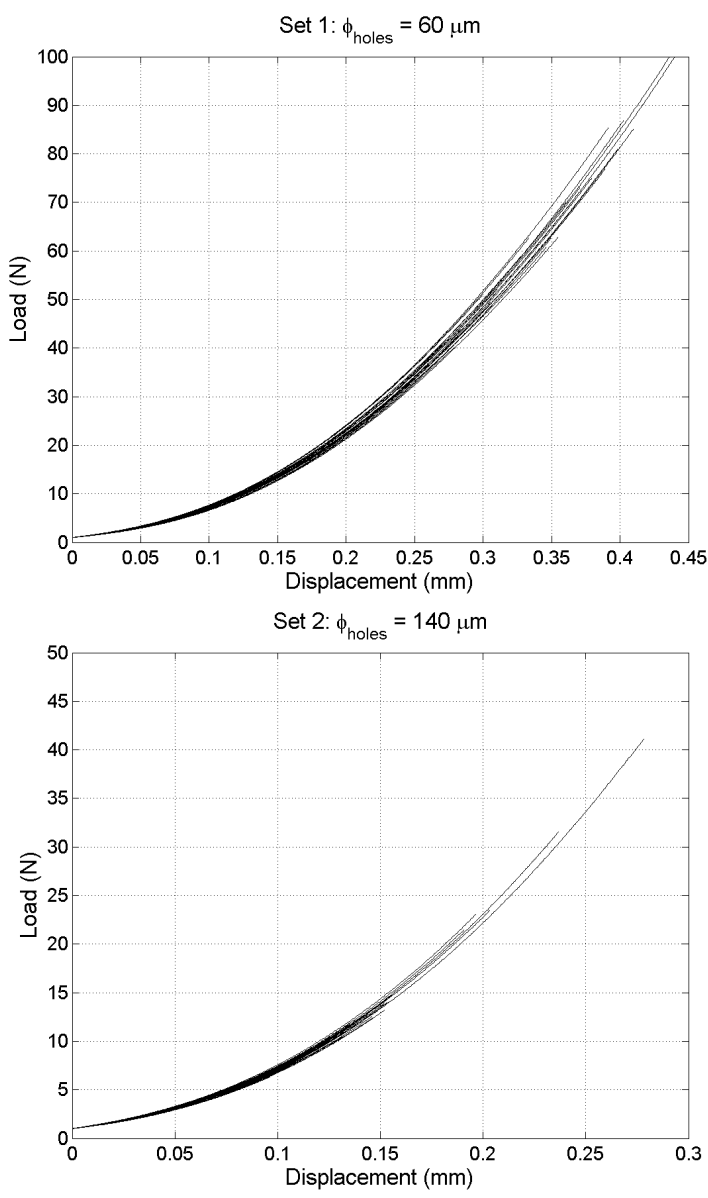

Fig. 5. Ring on Ring test results for sets 1 and 2. 
In Figure 5, the non linear behavior of the samples during the test is clearly shown. This behavior is due to the large displacements observed in the tests and the contact between the samples and the rings. Therefore, analytical methods are not valid to calculate the maximum stress in the moment of failure because they are based in a linear behavior of the samples. The Finite Element Method has been used to evaluate the stress state of the samples in the moment of failure. This method makes possible to simulate the tests considering the non linearities described above and including the special features of the material, i.e. the anisotropic behavior of the silicon crystal.

Wafer and supports are modeled with shell elements. In previous studies [8] it was demonstrated that the influence of the holes in the stiffness of the wafer can be neglected. Therefore, two models without holes are developed: one model corresponding to the thinnest wafer and other model corresponding o the thickest one. Test result and the models developed can be seen in figure 6 .

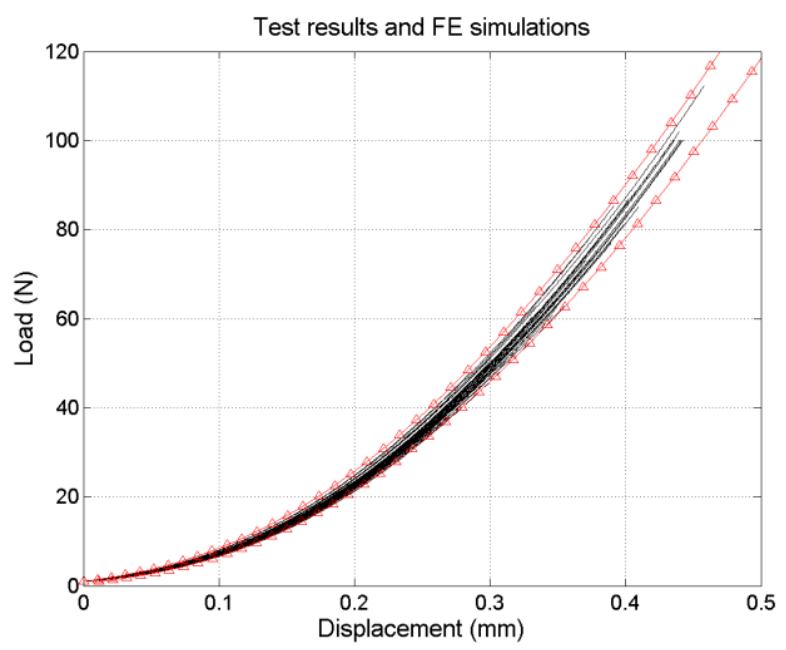

Fig. 6. Test results and FE simulations

The fracture stress for all samples is obtained through a linear interpolation taking into account the elastic energy stored in the wafer before failure and its thickness. It is considered the maximum principal stress is the failure stress.

The FE model is developed using the commercial package ANSYS. When the loading of a wafer is simulated, the stress distribution on it is obtained.

\section{Statistical Analysis of the MeAsures}

Once the fracture stress of the wafers is obtained, the resulting values for each set are fitted to a three parameter Weibull distribution by the least square method. This cumulative distribution function is expressed as follows [9].

$$
P_{f}(\sigma)=1-\exp \left[-\int_{A \mid \sigma>\lambda}\left(\frac{\sigma-\lambda}{\delta}\right)^{\beta} d A\right]
$$

Where $\lambda$ is the location parameter representing a threshold stress, $\delta$ is the scale parameter and $\beta$ is the shape parameter which gives information about the scattering of the samples. This function depends on the size of the loaded area. This dependence is known as the size effect and it takes into account that greater size of the sample implies lower strength values. The size effect correction requires an iterative procedure [10] that includes the calculation of the equivalent area of each test.

In this case, since all samples and tests have the same dimensions, it has been supposed that the equivalent area is similar. As the strength comparison between sets is more important in this study than the actual value of the strength, the equivalent area has been considered as unity giving the next expression for the probability of failure:

$$
P_{f}(\sigma)=1-\exp \left[-\left(\frac{\sigma-\lambda}{\delta}\right)^{\beta}\right]
$$

In this case, the scale parameter has the same dimensions as $\sigma$ and the sum of the scale and location parameters gives the characteristic fracture stress $\sigma_{\theta}$ which counts on the $63.2 \%$ of the samples will fail. This value is employed to characterize the strength of each set.

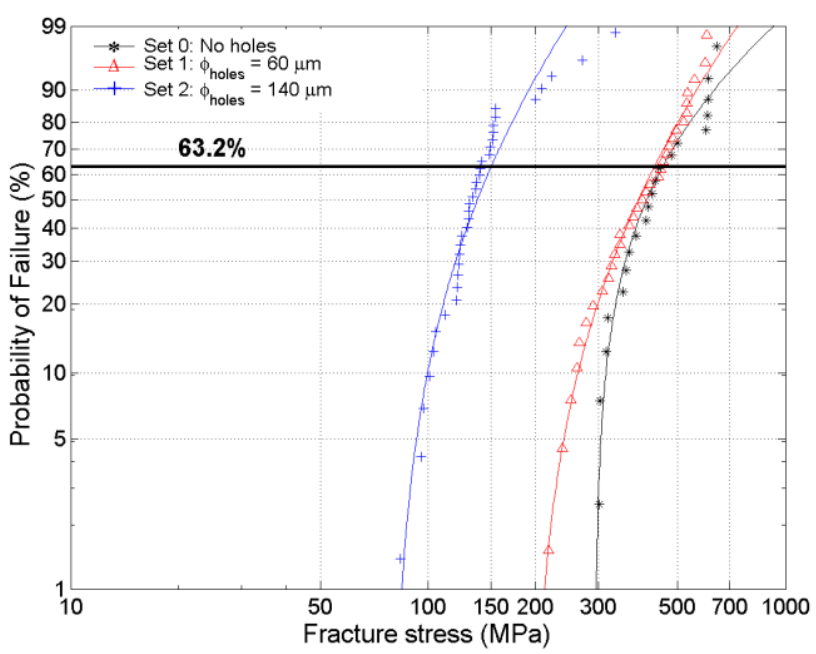

Fig. 7. Test results and FE simulations

Results of the fitting are shown in figure 7 and summarize in table I. 


\section{RESULTS}

The analysis of the Weibull parameters for each set show that the presence of holes affects to the mechanical strength of the wafers.

\begin{tabular}{|l|cccc|}
\cline { 2 - 5 } \multicolumn{1}{c|}{} & $\boldsymbol{\lambda}(\mathbf{M P a})$ & $\delta(\mathbf{M P a})$ & $\boldsymbol{\beta}$ & $\sigma_{\theta}(\mathbf{M P a})$ \\
\hline Set 0 & 293.12 & 160.12 & 1.11 & 453.24 \\
Set 1 & 191.55 & 237.97 & 1.86 & 429.52 \\
Set 2 & 78.72 & 71.45 & 1.83 & 150.16 \\
\hline
\end{tabular}

Table I. Results of Weibull adjustment.

Regarding to the characteristic fracture stress, it can be seen that the tiny holes of set 1 don't have too much influence in the mechanical strength. Previous results $[1,3,4,8]$ show a major reduction due to the presence of holes with similar diameter but in these cases, the distance between holes was much smaller. In those studies, the samples prepared represented the physical structure of wafers for EWT solar cells where holes are closer than those present in the samples prepare for this study. Therefore, there was an overlapping between the zones affected by the presence of holes reducing more significantly the mechanical strength of the samples. Moreover, it can be seen in Table I that the mechanical strength of set 2 is clearly affected by the holes of diameters of $140 \mu \mathrm{m}$. In this case, even though the distance between holes prevents the overlapping described above, the diameter of the holes is so high that the stress concentration around them reduces considerably the strength of the samples.

Furthermore, the comparison of the shape parameter of each set shows that the scattering of set 0 is higher than the other sets since $\beta$ has a lower value. Results of sets 1 and 2 are similar scattered. For set 0 , the study is estimating the Weibull parameters of wafers without holes being micro-cracks or defects in the structure the cause of the failure. Therefore, the scattering is higher. However, for sets 1 and 2, the failure is produced by the stress concentration around the holes reducing the scattering of the test results.

\section{SUMMARY AND CONCLUSION}

A methodology for analyzing the mechanical strength of drilled wafers as the EWT and MWT has been established, and the influence of different hole sizes has been studied.

With this aim, three sets of wafers have been prepared. The first set has no holes, the second one has holes with a diameter of $60 \mu \mathrm{m}$ and the last one has holes with a diameter of $140 \mu \mathrm{m}$.

All the samples have been tested by the Ring on Ring bending test and a numerical model has been developed in order to get the stress distribution on the samples just before the failure. Finally, a statistical study has been carried out by fitting the failure stresses to a three parameter Weibull distribution.
As it was expected, it has been observed that the presence of holes in the wafer reduces its the mechanical strength, and as the hole diameter increases the wafer becomes weaker. Also, it has been observed that the etching process after drilling step can remove the laser damage almost completely. For samples with small holes, mechanical strength can reach values close to mechanical strength of no drilled samples.

\section{ACKNOWLEDGEMENT}

This work has been made under the sponsorship of the Research, Development and Innovation State Secretary, belonging to Spanish Ministry of Economy and Competitiveness, in its contracts TEC2008-06798-C03 and TEC2011-28423-C03.

\section{REFERENCES}

[1] E. Cereceda, J. Barredo, J. R. Gutierrez, J. C. Jimeno, "Mechanical Stability in Crystalline Silicon Solar Cells", in Proceedings of the 25th European Photovoltaic Solar Energy Conference, Valencia, Spain, 2010, pp. 1665 - 1668.

[2] S. Schoenfelder, M. Oswald, C. Fischer, H.-U. Zühlke, C. Berbig, T. Geppert, T. Wütherich, H.-J. Krokoszinski, J. Bagdahn, "Investigations of Laser and Design Parameters of Via Holes on Mechanical Strength of Metal Wrap through Solar Cells", in Proceedings of the 25th European Photovoltaic Solar Energy Conference, Valencia, Spain, 2010, pp. 2578-2583.

[3] G. Cueto-Felgueroso, J. Barredo, E. Cereceda, y L. Hermanns, "Study of the Mechanical Strength Improvement of Wafers for EWT Solar Cells by Chemical Etching After the Drilling Process", in Proceedings of the 26th European Photovoltaic Solar Energy Conference, Hamburg, Germany, 2011, pp. 1589 1592.

[4] J. Barredo, L. Hermanns, I. del Rey, A. Fraile, E. Alarcón, "Optimizing the Density of Holes of EWT Solar Cells Taking into Consideration Mechanical Aspects", in Proceedings of the 26th European Photovoltaic Solar Energy Conference, Hamburg, Germany, 2011, pp. 1585 - 1588.

[5] R. Koepge, S. Schoenfelder, T. Giesen, C. Fischmann, A. Verl,J. Bagdahn, "The Influence of Transport Operations on the Wafer Strength and Breakage Rate" in Proceedings of the 26th European Photovoltaic Solar Energy Conference, Hamburg, Germany, 2011, pp. 2072-2077

[6] B. Micciche, B. Dingle, C. Meisser, "Understanding the Causes for Cell Breakage During the Cell Interconnecting Process- Part I", in Proceedings of the 21st European Photovoltaic Solar Energy Conference, Dresden, Germany, 2006, pp. 2128-2132.

[7] S. Schönfelder, A. Bohne, J. Bagdahn, "Comparison of test methods for strength characterization of thin solar wafer", in Proceedings of the 22nd European Photovoltaic Solar Energy Conference, Milan, Italy, 2007, pp. 1636-1640.

[8] J. Barredo, A. Fraile, I. del Rey, L. Hermanns. "The Influence of holes in the mechanical properties of EWT solar cells", in the $11^{\text {th }}$ International Conference on Mechanical Behaviour of Materials, Com Lake, Italy, 2011.

[9] W. Weibull, "A statistical theory of the strength of materials", Proceedings of the Royal Swedish Institute of Engineering Research, Nr.151, 1939.

[10] C. Przybilla, A. Fernández-Canteli, E. Castillo, "Deriving the primary cumulative distribution function of fracture stress for 
